\title{
Target Recognition in ISAR Images based on Relative Phases of Complex Wavelet Coefficients and Sparse Classification
}

Conference Paper · March 2018

CITATIONS

0

4 authors:

\section{Ayoub Karine}

ENSTA Bretagne

11 PUBLICATIONS 7 CITATIONS

SEE PROFILE

Ali Khenchaf

ENSTA Bretagne

309 PUBLICATIONS 1,172 CITATIONS

SEE PROFILE
READS

49



Abdelmalek Toumi

ENSTA Bretagne

55 PUBLICATIONS 144 CITATIONS

SEE PROFILE

Mohammed El Hassouni

Mohammed V University in Rabat

92 PUBLICATIONS 236 CITATIONS

SEE PROFILE

Some of the authors of this publication are also working on these related projects: 


\title{
Target Recognition in ISAR Images based on Relative Phases of Complex Wavelet Coefficients and Sparse Classification
}

\author{
Ayoub Karine ${ }^{1,2}$, Abdelmalek Toumi ${ }^{1}$, Ali Khenchaf ${ }^{1}$, Mohammed El Hassouni ${ }^{2,3}$ \\ ${ }^{1}$ Lab-STICC UMR CNRS 6285, ENSTA Bretagne, Brest, France \\ \{abdelmalek.toumi, ali.khenchaf\}@ensta-bretagne.fr \\ ${ }^{2}$ LRIT-CNRST, URAC 29, Rabat IT Center, Faculty of Sciences, Mohammed V University in Rabat, Rabat, Morocco \\ \{ayoub.karine\}@gmail.com \\ ${ }^{3}$ LRIT-CNRST, URAC 29, Rabat IT Center, FLSH, Mohammed V University in Rabat, Rabat, Morocco \\ \{mohamed.elhassouni\}@gmail.com
}

\begin{abstract}
In this paper, we present a novel approach for radar automatic target recognition on inverse synthetic aperture radar (ISAR). This approach is composed by two complementary steps: feature extraction and recognition. For the feature extraction step, we adopt a statistical modeling of the ISAR image in the complex wavelet domain. For doing so, we apply the dual-tree complex wavelet transform (DT-CWT) for each ISAR image in the database. After that, the relative phases of the resulting complex coefficients are computed. These relative phases are after statistically modeled using the Von Mises distribution. The estimated statistical parameters compose the feature vector of the ISAR images. Regarding to the recognition rate, the constructed feature vector is fed into the sparse representation based classification (SRC). More precisely, the training feature vectors are used as the atoms of a dictionary. The test feature vector is recognized according to its sparse linear combination with the dictionary. Extensive experiments and comparisons with other methods on ISAR images database demonstrate the effectiveness of the proposed approach.
\end{abstract}

Keywords-Automatic target recognition, inverse synthetic aperture radar, relative phase modeling, sparse representation

\section{INTRODUCTION}

Automatic target recognition (ATR) using radar images is an ongoing research in several military and civilian fields. The ATR system aims to recognize radar targets based on various image and signal processing techniques. Among the exploited radar images in the literature, we distinguish the inverse synthetic aperture radar (ISAR) images. These latter are acquired by the motion of the targets and the electromagnetic scattering principle. Generally speaking, the ATR systems based on ISAR images include three complementary steps [1]: detection, discrimination and recognition. We focus in this paper to the two last steps.

Regarding to the feature extraction (discrimination), several methods are used in the literature to describe the ISAR images such as polar representation [1, 2, 3], Fourier descriptor [4] and the combination of the wavelet transform and local binary pattern [5]. Recently, the statistical modeling of the image in the complex wavelet domain has demonstrated its performance in different image processing fields such as image retrieval $[6,7]$, image classification [8] and target recognition in radar images [9, 10]. Generally, this approach of image characterization has three degree of freedoms: the choice of the complex wavelet decomposition, the type of the extraction information and the statistical models. This latter depends on the information extracted of the complex wavelet coefficients. Among these informations, we cite the magnitude, the relative phase, the real part and the imaginary part. In this work, we give a special attention to the relative phase information extracted from the DT-CWT coefficients. The relative phases are practically modeled in the literature using the circular models such as the Von Mises [7]. According to this model, we estimate the two statistical parameters for the relative phases of each complex subband. The concatenation of these statistical parameters for all produced complex subband leads to construct the feature vector of the ISAR image.

For the recognition task, many classification methods are exploited by the researchers for radar target recognition. We cite the K-nearest neighbor (KNN) [5, 4, 11], the support vector machine (SVM) [12] and the combination of these two classifiers with the neural network (NN) method [13]. Recently, the sparse representation based classification [14] method has proved its performance in different pattern recognition applications and it is extended also to the ATR systems based on synthetic aperture radar (SAR) images $[15,16]$ and ISAR images $[9,10]$. We investigate in this paper the use of the relative phases statistical parameters in the complex wavelet domain as an input of the SRC classifier. The overall scheme of the proposed method is illustrated in Figure 1.

The remainder of this paper is organized as follows. We describe in section II the steps to extract the feature vectors from ISAR images. In section III, we give an overview of the SRC method. The experiment results are carried out in section IV to evaluate the proposed approach. Finally, the conclusion is provided in section $\mathrm{V}$.

\section{RELATIVE PHASE MODELING AND FEATURE EXTRACTION}

\section{A. Complex wavelet decomposition}

In this work, we adopt the DT-CWT method [17] in order to generate the complex wavelet coefficients. This method is 


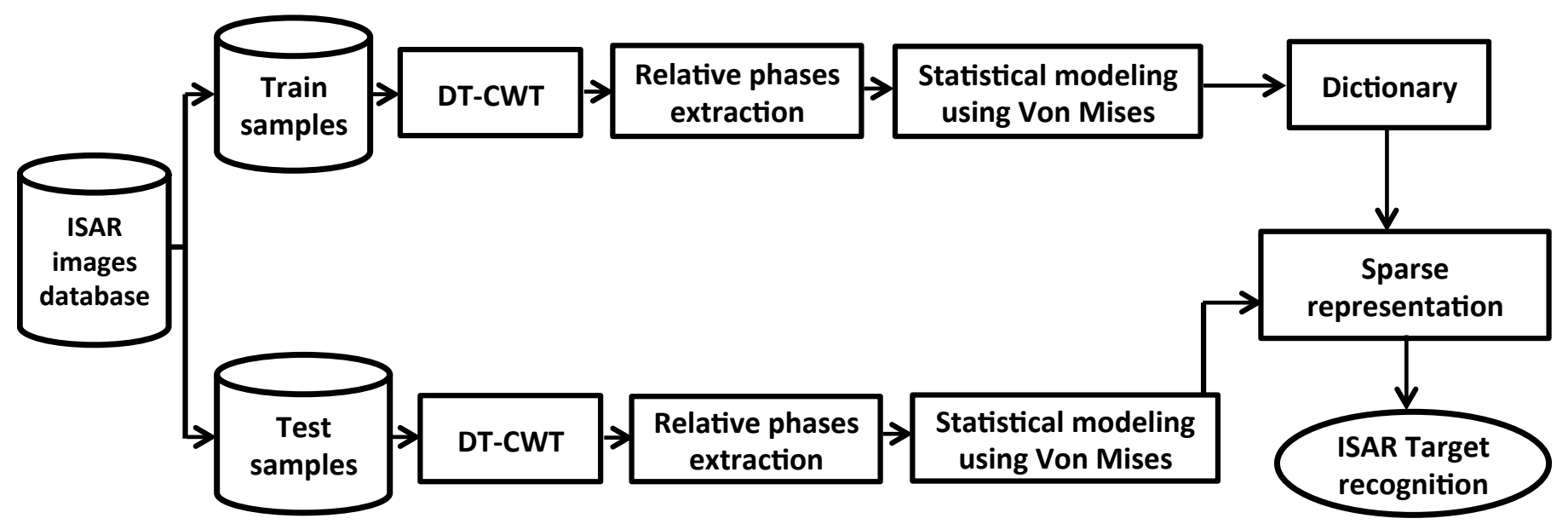

Fig. 1. Flowchart of the proposed approach.

proposed by Kingsbyry to overcome the limit of the discrete wavelet transform (DWT) which is the reduced number of generated subbands (orientations). For a given level of decomposition, this method produces six subbands of six orientations : $\left(+15^{\circ},+45^{\circ},+75^{\circ},-75^{\circ},-45^{\circ}\right.$ and $\left.-15^{\circ}\right)$. Each subband is composed by different complex numbers and it is shift invariant. We study in this paper the relative phases of these complex numbers [18]. From each complex numbers $z=a i+b$, the phase $\phi$ can be calculated as follows:

$$
\begin{aligned}
\phi & =\arctan \left(\frac{\operatorname{Im}(z)}{\operatorname{Re}(z)}\right) \\
& =\arctan \left(\frac{a}{b}\right)
\end{aligned}
$$

Then the relative phase is computed as follows:

$$
\theta=\phi_{\mathrm{SB}_{1}}-\phi_{\mathrm{SB}_{2}}
$$

where $\phi_{\mathrm{SB}_{1}}$ and $\phi_{\mathrm{SB}_{2}}$ are the phases of two adjacent complex subbands.

\section{B. Statistical parameters estimation}

In order to capture the statistic behaviors of the relative phases in the complex wavelet domain, we propose to use the Von Mises distribution. The probability density function (pdf) of this distribution is described as follows:

$$
f(\theta ; \mu, v)=\frac{\mathrm{e}^{v \cos (\theta-\mu)}}{2 \pi \mathrm{I}_{0}(v)}
$$

with

- $\theta$ is the angular random variable which is the relative phase in our case

- $\quad \mu$ represents the mean direction

- $\quad v$ denotes the concentration parameter

- $\mathrm{I}_{0}=\frac{1}{\pi} \int_{0}^{\pi} \mathrm{e}^{v \cos \theta} d \theta$ is the modified Bessel function

The Von Mises parameters ( $\mu$ and $v$ ) are estimated according to the maximum likelihood methods [7].
Finally, the feature vector FV of the ISAR images is composed by the Von Mises parameters in the complex wavelet domain:

$$
\mathrm{FV}=\left[\mu_{i}, v_{i}\right]_{1 \leq i \leq 6 J}
$$

where $J$ is the number of scales and $i$ the index of the complex subbands.

\section{RADAR TARGET RECOGNITION}

\section{A. Sparse representation based classification}

In this work, we propose to recognize the feature vector constructed in the previous step (Equation 4) using the SRC method. The first step of this method is the construction of a dictionary $A$. Each atom (column) of the dictionary is composed by the feature vectors (the Von Mises parameters) FV of the training set:

$$
A=\left[\mathrm{FV}_{1}, \ldots, \mathrm{FV}_{n}\right] \in \mathbb{R}^{m \times n}
$$

where $n$ is the size of training radar images and $m$ is the dimension of the feature vector which equals to $2 \times 6 \times J$. This method classifies a test feature vector $y$ based on its sparse linear representation from the dictionary $A$ :

$$
y=A x
$$

with $x \in \mathbb{R}^{n}$ is a sparse vector. To obtain this vector, the following optimization problem should be solved:

$$
\hat{x}=\min _{x}\|x\|_{1} \text { subject to }\|y-A x\|_{2} \leq \epsilon
$$

where $\|x\|_{1}=\sqrt{\sum_{i=1}^{n}\left|x_{i}\right|}$ denotes the $l^{1}$-norm and $\epsilon$ is the allowed error tolerance. After the finding of the optimal solution $\hat{x}$, a characteristic function $\delta_{i}: \mathbb{R}^{n} \rightarrow \mathbb{R}^{n}$ is computed that have all values to be zero except the coefficients corresponding to class $i$. Finally, the class of the test sample is the class that has the minimum reconstruction error:

$$
\begin{aligned}
\operatorname{class}(y) & =\min _{i} r_{i}(y) \\
& =\min _{i}\left\|y-\hat{y}_{i}\right\|_{2} \\
& =\min _{i}\left\|y-A \delta_{i}(\hat{x})\right\|_{2}
\end{aligned}
$$




\section{EXPERIMENTAL RESULTS}

\section{A. Database description}

In order to assess the performance of the proposed approach, we use ISAR images database. These radar images are acquired in an anechoic chamber using twelve reducedscale (1/48) aircrafts (classes): Rafale, Harrier, F104, Tornado, A10, F117, F16, MIG29, F14, F18, F4 and F15. Each class is composed by 162 ISAR images of $256 \times 256$ grayscal pixels. To reconstruct theme, the the inverse fast Fourier transform (IFFT) method [1] is adopted. Examples of the ISAR images contained in this database as well as its ground truth are illustrated in Figure 2.
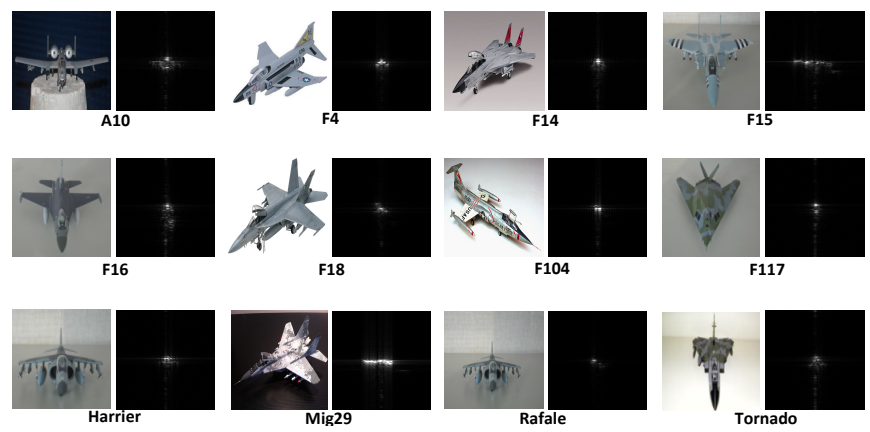

Fig. 2. Description of ISAR images database composed by twelve classes (aircraft targets). Beside each ISAR image, we display its ground truth.

\section{B. Radar target recognition results}

For each ISAR image in the database, we compute the feature vector composed by $6 \times J \times 2$ statistical parameters, where $J$ is the scale of the decomposition. After that, we conduct the hold-out cross validation in order to split the database to training and testing samples. For this end, we use several quotients of the database less than $50 \%$ to construct the training samples features. The remaining features vectors are considered as test samples. The training features are stacked together to build the dictionary. In the other words, the quotient of training samples affects the number of atom in the dictionary. For instance, by considering $40 \%$ of the database for train and remaining for test, we have $65 \times 12=780$ atoms in the dictionary (780 images in the training database) and $1944-780=1164$ images for testing. In order to make a fair comparison, the recognition rates are compared with two classification methods: KNN and SVM. We underline that we use the same descriptor for the different classifiers. The parameters of these classifiers are tuned empirically. Considering $\mathrm{KNN}$, the number of neighbor equals to $5(K=5)$ is considered. Regarding to SVM, the linear function is used.

We record in the Table $\mathrm{I}$, the comparison of different methods in term of recognition rates. From this table, many observations can be drawn:

- All classifiers are sensitives to the quotient of training samples (number of atoms in the dictionary). When this quotient increases, the recognition performance rise as well. For example, considering 3 levels of decomposition and SRC, the accuracies are $70.21 \%$, $77.11 \%, 82.50 \%$ and $87.99 \%$ respectively for the quotients $10 \%, 20 \%, 30 \%$ and $40 \%$.
- The increasing of the level of decomposition improves systematically the recognition rates. We underline here that three levels denote the concatenation of the feature vectors of the first, second and three levels of decomposition. For instance, by adopting a quotient of $40 \%$, the recognition rates for SRC in one level, two levels and three levels are respectively $54.80 \%$, $77.19 \%$ and $87.99 \%$. Then, we have an improvement of $33,13 \%$ between the first and the last levels.

- By considering the third levels and $40 \%$ of database for training, the three classifiers yield recognition rates grater than $74 \%$. This result demonstrate that the statistical modeling of ISAR images phases in the complex wavelet domain is a suitable approach to describe the content of the ISAR images.

- The SRC method outperforms the KNN and SVM classifiers for all levels of decomposition and under all quotients of training samples. For example, in the third level and under the quotient $40 \%$, the recognition rates of the $\mathrm{KNN}, \mathrm{SVM}$ and $\mathrm{SRC}$ are respectively $78.64 \%, 74.36 \%$ and $87.99 \%$. Then the SRC are $9.35 \%$ and $13.63 \%$ better in average than KNN and SVM. This improvement demonstrate the power of the combination of the proposed descriptor and the sparse classifier to recognize the ISAR images.

We illustrate in Figure 3 the confusion matrix using the proposed descriptor with the three classifiers: KNN, SVM and SRC. These confusion matrix is obtained on three level of decomposition and using $40 \%$ as a quotient of the training samples. The diagonal values of each confusion matrix represent the recognition rate per class which should be high, while the other values provides the misrecognition rate which must be low. The high misrecognition rate is given by SVM with $23.7 \%$ followed by KNN with $22.7 \%$ and SRC with $17.5 \%$. For all ISAR images classes, the SRC leads to a recognition rate grater than $68 \%$. The low recognition rate are provided by F4 target for all classifiers. This target is generally confused with Mig29 one that is due to the high similarity between its ground truth (see Figure 2). The SRC classifier provides the high overall recognition rates despite that it doesn't achieve the high recognition rate for all classes.

\section{Conclusion}

We have investigated in this paper the use of the classification via sparse representation in the complex wavelet domain to recognize the aircraft targets on ISAR images. According to a statistical approach, the feature vector is constructed by modeling the ISAR image in the complex wavelet domain. For this end, the relative phases of the complex subband are modeled using the Von Mises and the estimated parameters are stacked together to build the feature vector. To reach the recognition, the spare representation strategy are adopted. Extensive empirical comparisons are conducted with the KNN, SVM in ISAR image database. The simulation results show that the probabilistic properties of the ISAR images phases in the complex wavelet domain can achieve better performance especially combined by the SRC method. As future works, we aim to apply our approach on different radar images databases as well as the investigation of other statistical models. 
TABLE I. RECOGNITION RATES (\%) OF DIFFERENT METHODS UNDER DIFFERENT QUOTIENT OF TRAINING SAMPLES.

\begin{tabular}{|c|c|c|c|c|c|c|c|c|c|c|c|c|}
\hline & \multicolumn{12}{|c|}{ Levels of decomposition } \\
\hline & & & & & & & & & & & & \\
\hline Quotient of training samples & $10 \%$ & $20 \%$ & $30 \%$ & $40 \%$ & $10 \%$ & $20 \%$ & $30 \%$ & $40 \%$ & $10 \%$ & $20 \%$ & $30 \%$ & $40 \%$ \\
\hline KNN & 43.57 & 50.42 & 53.60 & 57.29 & 58.49 & 69.58 & 74.78 & 75.39 & 61.92 & 60.26 & 75.15 & 78.64 \\
\hline SVM & 28.13 & 32.03 & 36.47 & 39.19 & 50.83 & 58.65 & 59.85 & 62.26 & 55.75 & 66.82 & 71.76 & 74.36 \\
\hline SRC & 46.48 & 53.44 & 53.68 & 54.80 & 67.75 & 72.67 & 77.43 & 77.19 & 70.21 & 77.17 & 82.50 & 87.99 \\
\hline
\end{tabular}
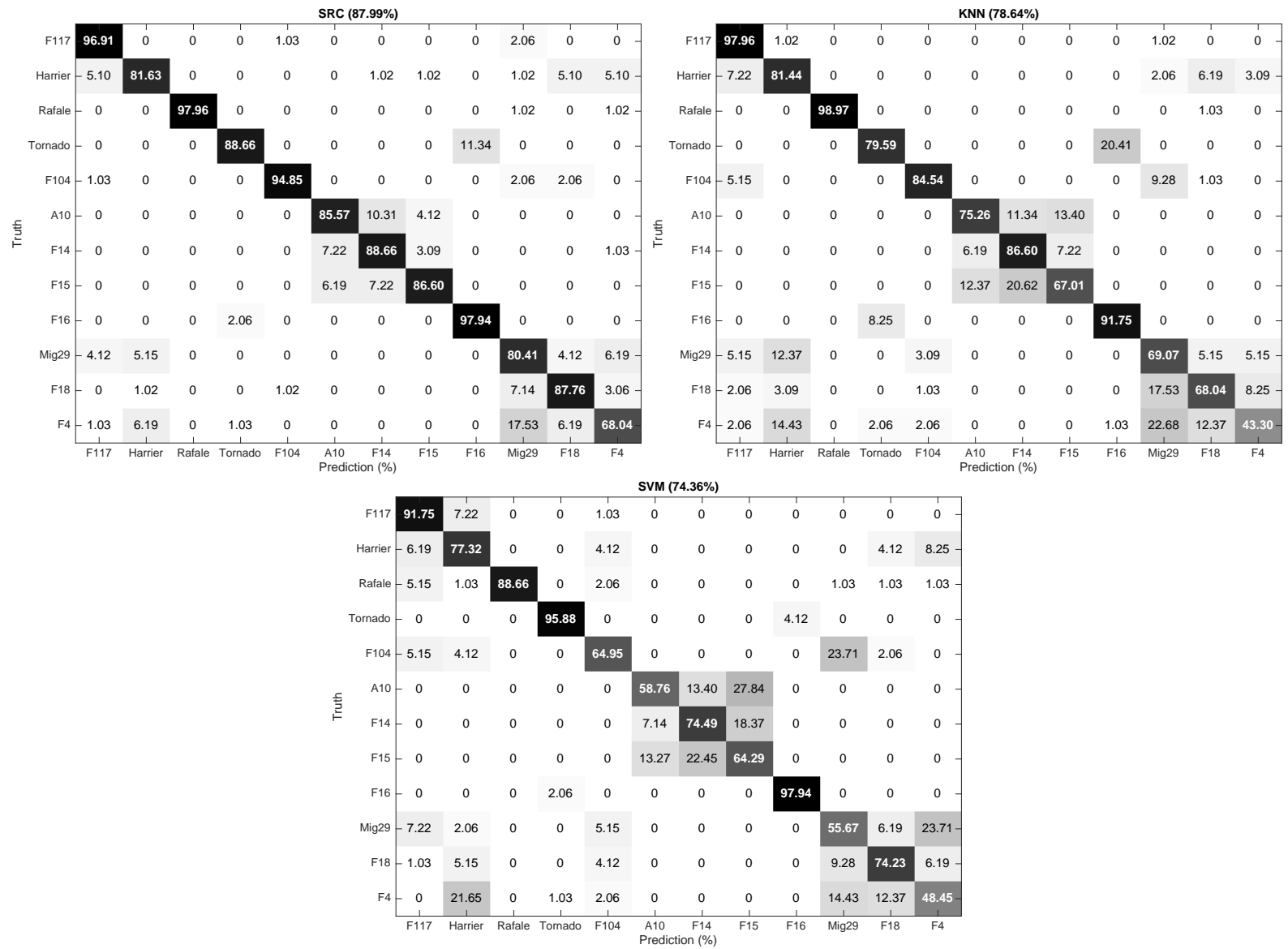

Fig. 3. Confusion matrix of different methods: SRC, KNN and SVM.

\section{REFERENCES}

[1] A. Toumi, A. Khenchaf, and B. Hoeltzener, "A retrieval system from inverse synthetic aperture radar images: Application to radar target recognition," Information Sciences, vol. 196, pp. 73-96, 2012.

[2] A. Toumi and A. Khenchaf, "Log-polar and polar image for recognition targets," in IEEE International Geoscience and Remote Sensing Symposium (IGARSS), July 2010, pp. 1609-1612.

[3] K. Kim, D. Seo, and H. Kim, "Efficient classification of ISAR images," IEEE Transactions on Antennas and Propagation, vol. 53, no. 5, pp. 1611-1621, 2005.

[4] B. Belkhaoui, A. Toumi, A. Khalfallah, A. Khenchaf, and M. S. Bouhlel, "Fusion fourier descriptors from the EM, $\mathrm{k}$-means and fisher algorithms for radar target recognition," International Journal of Computer and Information Technology, vol. 2, no. 4, 2013.

[5] F. Wang, W. Sheng, X. Ma, and H. Wang, "Target automatic recognition based on ISAR image with wavelet transform and mblbp," in International Symposium on
Signals, Systems and Electronics, vol. 2, Sept 2010, pp. $1-4$.

[6] R. Kwitt and A. Uhl, "Lightweight probabilistic texture retrieval," Image Processing, IEEE Transactions on, vol. 19, no. 1, pp. 241-253, 2010.

[7] A. Vo and S. Oraintara, "A study of relative phase in complex wavelet domain: Property, statistics and applications in texture image retrieval and segmentation," Signal Processing: Image Communication, vol. 25, no. 1, pp. 28-46, 2010.

[8] H. Oulhaj, M. Rziza, A. Amine, H. Toumi, E. Lespessailles, R. Jennane, and M. E. Hassouni, "Trabecular bone characterization using circular parametric models," Biomed. Signal Proc. and Control, vol. 33, pp. 411-421, 2017.

[9] A. Karine, A. Toumi, A. Khenchaf, and M. El Hassouni, "Aircraft recognition using a statistical model and sparse representation," in Proceedings of the International Conference on Big Data and Advanced Wireless Technologies (BDAW), Blagoevgrad, Bulgaria, 2016. 
[10] A. Karine, A. Toumi, A. Khenchaf, and M. E. Hassouni, "Target recognition in radar images using weighted statistical dictionary-based sparse representation," IEEE Geosci. Remote Sensing Lett., vol. 14, no. 12, pp. 24032407, 2017.

[11] _ - "Saliency attention and sift keypoints combination for automatic target recognition on MSTAR dataset," in 2017 International Conference on Advanced Technologies for Signal and Image Processing (ATSIP), Fez, Morocco, May 22-24, 2017, 2017, pp. 1-5.

[12] _ - "Visual salient sift keypoints descriptors for automatic target recognition," in IEEE European Workshop on Visual Information Processing (EUVIP), Marseille, France, 2016.

[13] I. Jdey, A. Toumi, M. Dhibi, and A. Khenchaf, "The contribution of fusion techniques in the recognition systems of radar targets," in IET International Conference on Radar Systems (Radar), Oct 2012, pp. 1-5.

[14] J. Wright, A. Y. Yang, A. Ganesh, S. S. Sastry, and Y. Ma, "Robust face recognition via sparse representation," IEEE transactions on pattern analysis and machine intelligence, vol. 31, no. 2, pp. 210-227, 2009.

[15] G. Dong, G. Kuang, N. Wang, and W. Wang, "Classification via sparse representation of streerable wavelet frames on grassmann manifold: Application to target recognition in SAR image," IEEE Transactions on Image Processing, vol. 26, no. 6, pp. 2892-2904, 2017.

[16] X. Xing, K. Ji, H. Zou, W. Chen, and J. Sun, "Ship classification in TerraSAR-X images with feature space based sparse representation," IEEE Geoscience and Remote Sensing Letters, vol. 10, no. 6, pp. 1562-1566, 2013.

[17] N. G. Kingsbury, "Complex wavelets for shift invariant analysis and filtering of signals," Applied and computational harmonic analysis, vol. 10, no. 3, pp. 234-253, 2001.

[18] Y. Rakvongthai and S. Oraintara, "Statistical texture retrieval in noise using complex wavelets," Signal Processing: Image Communication, vol. 28, no. 10, pp. 1494 $-1505,2013$. 